\title{
Nuevas Configuraciones Económicas en el Asia-Pacífico y sus Consecuencias para América Latina: Desde el APEC a la Alianza del Pacífico
}

\section{Isabel Rodríguez Aranda}

Pesquisadora do Centro de Estudios de Relaciones Internacionales (CERI) e diretora do curso de Ciência Política e Políticas Públicas, da Facultad de Gobierno, Universidad del Desarrollo, Santiago de Chile. E-mail: isabelrodriguez@udd.cl.

\section{INTRODUCCIÓN}

— ste artículo se propone analizar - a través de los conceptos de re¿ gionalización, regionalismo e integración - las nuevas tendencias en la configuración de los espacios de cooperación económica en la región del Asia-Pacífico. Desde la Cumbre de Santiago de Chile en 2004, los líderes del Foro de Cooperación del Asia-Pacífico (APEC, por su sigla en inglés) pasan a aceptar que la estrategia de liberalización unilateral, practicada desde 1989, daría paso a una liberalización formal por medio de la firma de tratados de libre comercio (TLC), siendo el objetivo en el mediano plazo - año 2020 según las metas de Bogor ${ }^{1}$ - alcanzar un área de libre comercio total.

A partir de fines de la década de los 1980 e inicios de los 1990, comienza una tendencia generalizada en el sistema internacional a seguir los principios de la liberalización económica como herramienta para lograr crecimiento económico y desarrollo. Esta tendencia se concreta por la implementación de distintos instrumentos que permitan avanzar en dicha liberalización: desde la liberalización unilateral y tratados de libre comercio hasta la formación de bloques con acuerdos más profundos, como las uniones aduaneras, mercados comunes y uniones monetarias. Estos instrumentos darán origen a diversos bloques ecoDADOS - Revista de Ciências Sociais, Rio de Janeiro, vol. 57, n-2, 2014, pp. 553 a 580. 
nómicos de países que van a configurar el sistema internacional, que, en palabras de Ibáñez (2000:1-2), se describe como una "oleada de integración regional" a nivel global formadora de bloques y, según de Di Filippo y Franco (2000:60), como un preferencialismo que crea comercio entre los miembros de un bloque sin colocar barreras a los países no miembros. En el caso de las áreas de libre comercio, tenemos, por ejemplo, la creación del Área de Libre Comercio de América del Norte (Nafta, por su sigla en inglés) el año 1991, el Área de Libre Comercio de la Asean ${ }^{2}$, en 1992, y, en el caso de bloques de integración profunda, los avances en la Unión Europea (UE) con el Acta Única Europea, en 1987, y el Tratado de Maastricht, en 1991, las experiencias latinoamericanas, como el Mercado Común del Sur (Mercosur) de 1991, y otras ya existentes que se reformulan, como la Comunidad Andina (CAN) - ex Pacto Andino-, el Mercado Común Centroamericano (MCCA) y la Comunidad del Caribe (Caricom). No obstante, hay experiencias no vinculantes, como el APEC, creado en 1989, que también generan un bloque de cooperación y concertación económica para liberalizar las economías, pero sin firmar un tratado de libre comercio, y complementan esta tendencia global formando, en este caso, una región económica que llegará a abarcar a veintiún países o economías.

De este modo, será en este contexto económico internacional, amparado, legitimado e impulsado por el GATT (luego Organización Mundial de Comercio-OMC, en 1995), en que surge el APEC, cuya dinámica de integración económica no seguirá los parámetros de integración formal dictada por esta oleada de regionalismo abierto de bloques impulsado por tratados (López Aymes, 2002). Por el contrario, la región del Asia-Pacífico se conformará por un proceso de regionalización espontáneo, dinamizado principalmente por empresarios y académicos que van a impulsar una tendencia de los gobiernos de la región a cooperar en lo económico, formalizando un proceso de regionalismo que, en la práctica, podemos conceptualizar como un regionalismo abierto unilateral, y que es distinto al regionalismo en Europa o América Latina (Rodríguez, 2005).

Para explicar esta diferencia con el regionalismo que se desarrolla en Europa y América Latina, comenzaremos por diferenciar los conceptos de regionalización y regionalismo. Entendemos regionalismo como el proceso de integración que deciden impulsar los Estados participantes a través de un proyecto que se formaliza en un tratado, el cual determina los objetivos de integración económica, que van desde 
un Área de Libre Comercio hasta una Unión Económica (Sanahuja, 2009). Esta dinámica de regionalismo es la que caracteriza la integración regional en América Latina, con acuerdos de integración que firman los Estados y que constituyen proyectos a realizar, pero que en la práctica "avanzan" en niveles menores de integración. Por ejemplo, el Mercosur, cuyo proyecto formalizado en un tratado es llegar a ser un Mercado Común, pero que en la práctica es un bloque subregional que avanza hacia una Unión Aduanera como etapa previa al Mercado Común.

La regionalización, por el contrario, corresponde a un proceso espontáneo de integración económica, donde los actores no estatales son los que principalmente impulsan una interdependencia económica entre los países de una región (Ibáñez, 2000). Este es el caso de la región del Asia-Pacífico, donde se produce una interdependencia económica espontánea dinamizada por el intercambio económico entre los países que crean una estructura comercial dinámica y redes de producción integradas. Por consiguiente, el APEC surge de un proceso de regionalización espontánea, aspecto que se refleja en la dinámica de membresía al foro, es decir, para ser miembro del APEC se requiere tener altos índices de intercambio comercial con los países de la región (Rodríguez, 2005). Es sólo esta condición la que ha regido la selección de miembros y explica la membresía de Chile, México y Perú. También, consideremos que esta regionalización fue impulsada por actores no estatales que formalizan su cooperación en los foros regionales que preceden al APEC, a saber, el Pacific Basin Economic Council y el Pacific Economic Cooperation Council, ambos vigentes hasta el día de hoy.

Asimismo, una de las características más destacadas del APEC ha sido su carácter informal, es decir, sin una estructura institucional rígida, donde no participan Estados sino economías, y no hay acuerdos o tratados firmados. Más bien, el APEC tiene una estructura dinámica, en la cual cada economía participa en igualdad de condiciones, las decisiones se toman por consenso y se transmiten en declaraciones oficiales, todo bajo una meta de liberalización unilateral concertada que se expresa en los cuatros principios fundantes: impulsar el crecimiento y desarrollo de la región, contribuir a una mayor liberalización de la economía mundial, reforzar los efectos positivos resultantes de la creciente interdependencia económica y reducir las barreras al comercio de bienes, servicios e inversiones (Armanet, Alamos y O'Shea, 1996). Estos principios del APEC reflejan esta dinámica de trabajo concerta- 
do, es decir, son principios amplios y abarcadores de distintos temas, desde la liberalización y facilitación de comercio e inversiones, hasta temas de cooperación en áreas tan variadas como educación, cultura y tecnología (Rodríguez, 2005).

En consecuencia, el APEC consiste en un instrumento para el proceso de liberalización económica unilateral concertada, que podemos conceptualizar como regionalismo abierto unilateral, que se ejecuta con las siguientes dinámicas no excluyentes entre sí: liberalización unilateral; compromiso de extender la reducción de barreras a terceros países; extensión de la liberalización regional a terceros países sobre una base de reciprocidad, hasta que se instrumentalice la liberalización internamente, fundada en el principio de la nación más favorecida; y reconocimiento de que cualquier miembro del APEC puede extender la liberalización que haya acordado en ese marco a terceros países, sea en forma condicional o incondicional.

No obstante, desde el 2004 en adelante, el APEC ha iniciado una nueva etapa que busca impulsar la tendencia hacia un regionalismo formal y dinamizar la firma de tratados de libre comercio entre las economías de los países que integran el APEC, idea que se discutió en la Cumbre del APEC del año 2003, en Tailandia. Esta idea se formaliza en la Cumbre de Santiago realizada en Chile el año 2004, que reconoce la compatibilidad entre la formalización de tratados de libre comercio y las dinámicas de liberalización unilateral concertada. En la Declaración de Santiago, "Una Comunidad Nuestro Futuro", de la 12a Reunión de Líderes Económicos del APEC, de noviembre del 2004, se señala respecto a los Acuerdos Comerciales Regionales (en inglés, Regional Trading Arrangements o RTA) y los Acuerdos o Tratados de Libre Comercio (en inglés, Free Trade Agreements o FTA) que:

acordamos que estos juegan un rol constructivo en acelerar la liberalización en la región, contribuyendo así al logro de las metas de Bogor y al avance del proceso de la OMC. Para fortalecer esta contribución y asegurar acuerdos de estándares altos, nos complace aprobar las Buenas Prácticas para RTAs/FTAs en el APEC, las cuales son un significativo punto de referencia para los miembros del APEC en sus negociaciones de RTAs/FTAs. También nos comprometimos a una mayor transparencia en los RTAs/FTAs para facilitar la comprensión pública del alcance y efectos de estos acuerdos (Declaración de Santiago, 2004:2).

La consecuencia de lo anterior, y tesis central de este artículo, es que hay una nueva configuración de la región del Asia-Pacífico con un ni- 
vel intermedio de formalización, que se ha constituido con la creación de subregiones basadas en la firma de tratados de libre comercio tanto de carácter horizontal, es decir, entre países con similares niveles de desarrollo, como de tipo vertical, entre países con distintos niveles de desarrollo. De acuerdo a la conceptualización de Di Filippo y Franco (2000) sobre tipos de acuerdos de integración, se estarían suscribiendo en la actualidad en la región de Asia-Pacífico acuerdos de tipo A, es decir, acuerdos de libre comercio "que son áreas preferenciales de mercado orientadas a acelerar la vigencia en el plano regional de los principios del multilateralismo global en el marco de los criterios del así denominado regionalismo abierto" (Di Filippo y Franco, 2000:58). Estos acuerdos tipo A, según los autores mencionados, se diferencian de los acuerdos tipo B que "incluyen compromisos ubicados más allá de la esfera de los mercados y abarcan dimensiones políticas, sociales y culturales" (Di Filippo y Franco, 2000:59). Sin embargo, agregan los autores que esos compromisos políticos, sociales y culturales que afectan las políticas públicas de los países requieren compromisos jurídicamente vinculantes. Ese aspecto en el caso del APEC no estará presente hasta la Declaración de Santiago de 2004, dado que su dinámica era la de una cooperación unilateral concertada informalmente, que aun influyendo y aportando a las políticas públicas de los países en variados temas como medio ambiente, turismo, educación y recursos humanos, no generó un proceso jurídicamente vinculante. No obstante, el APEC ha pasado a una etapa vinculante desde 2004 aceptando la firma de acuerdos tipo $\mathbf{A}$ en espacios subregionales y con metas claras que apuntan a abarcar a toda la región del Asia-Pacífico.

Por lo tanto, esta dinámica de firmar acuerdos tipo A entre los países miembros del APEC nos permite identificar algunas tendencias nuevas en este foro que serán desarrolladas en este artículo. En primer lugar, y acorde al contexto económico internacional, hay una evolución del APEC que se expresa en la firma de acuerdos tipo A en el marco de la OMC. Segundo, con esta tendencia se produce una revitalización de los objetivos del APEC con el propósito de avanzar hacia la liberalización multilateral de comercio a través de la reciprocidad vinculante. Y, tercero, hay una dinámica nueva que tiende hacia una regionalización formal con miras a la posibilidad de crear un tratado de libre comercio entre las veintiuna economías y formar subregiones económicas que, desde 2004, se configuran en el Acuerdo Transpacífico de Asociación Económica Estratégica (P4), el Acuerdo de Asociación Transpacífico (TPP), el Área de Libre Comercio de la Asociación de Naciones del Su- 
deste Asiático con China, Japón y Corea del Sur (ASEAN+3), y la Alianza del Pacífico (AP).

Por otra parte, al analizar la participación de América Latina en estas nuevas configuraciones, concretamente a través de la subregión de la AP en la que participan Chile, Perú, México y Colombia, observamos un nuevo ingrediente, pues estos países latinoamericanos proponen firmar un acuerdo de tipo B - que busca crear un espacio económico donde circulen libremente todos los factores productivos, bienes y servicios, mano de obra y capital - para impulsar un proceso de integración que vaya más allá de un tratado de libre comercio.

La hipótesis que guía este artículo es que, desde el año 2004, en la región de Asia-Pacífico se ha impulsado una nueva configuración de relaciones económicas basada en la firma de tratados de libre comercio, creando subregiones estratégicas que se mueven en dos dinámicas: por un lado, una dinámica de cooperación que busca avanzar en función de la meta del APEC 2020, que consiste en formar un área de libre comercio regional en la Cuenca del Pacífico, y, por otro lado, dinámicas de rivalidad que permean estas subregiones en función de los intereses de las principales potencias de la región, como Estados Unidos y China. A su vez, en esta nueva configuración, los países participantes de América Latina, como Colombia, México, Perú y Chile, también entran en estas dinámicas. Su objetivo es buscar alianzas para ampliar mercados y relacionarse con estas principales economías a nivel global, y lo hacen construyendo la Alianza del Pacífico, una subregión de integración profunda que es más que un tratado de libre comercio. En esta tendencia, Chile ha tenido un rol protagónico al participar de las principales subregiones en formación, desempeño que se debe a la estrategia de inserción económica del país, que es pionera en la región latinoamericana en relacionarse con el Pacífico asiático por medio de tratados de libre comercio.

Los objetivos de este estudio son tres: primero, analizar el 2004 como un punto de formalización de una nueva etapa del APEC que abandona el modelo de regionalismo por liberalización unilateral concertada para tomar el camino de un regionalismo formal a través de un tratado de libre comercio regional que se plasma en las metas de Bogor 2020; segundo, demostrar que, en este proceso que se desarrolla desde el 2004, se han estructurado subregiones que protagonizan una nueva configuración regional que se ha formalizado en tratados de libre co- 
mercio - es el caso de la ASEAN+3, el P4, el TPP, y un acuerdo que es un proyecto de integración profunda, la AP-; y, tercero, determinar las estrategias de participación de los países miembros latinoamericanos en este espacio regional, en especial de Chile, para proyectar las oportunidades y desafíos que tienen en relación con la región y a las principales economías miembros, Estados Unidos y China.

\section{NUEVOS TIPOS DE PROCESOS SUBREGIONALES EN EL APEC: EL P4 Y EL TPP}

El Acuerdo Estratégico Transpacífico de Asociación Económica, denominado con las siglas P4, fue firmado el dieciocho de julio de 2005, en Nueva Zelanda, por este último país más Singapur, Brunei Darussalam y Chile, entrando en vigor el ocho de noviembre de 2006. El P4 surge justamente en el marco del APEC, en su cumbre del año 2002 - en México - y por iniciativa de tres países, Nueva Zelanda, Singapur y Chile, a los que se sumaría posteriormente Brunei Darussalam. Lo importante de este acuerdo en la nueva configuración de los espacios de cooperación económica en la región del Asia-Pacífico es que se constituye como el primer acuerdo trilateral que desde sus inicios fue inclusivo, ya que en lo inmediato aceptó un cuarto integrante (Brunei), y ha proyectado su ampliación negociando actualmente con cinco países: Australia, Estados Unidos, Perú, Vietnam y Malasia para formar el TPP, negociaciones que, al momento de este artículo, están en curso. Es el P4 el ejemplo paradigmático de la Declaración de Santiago en 2004, porque desde su formación trabaja por generar un área de libre comercio que se extienda por la región, en otras palabras, un acuerdo tipo A de carácter horizontal que, en definitiva, es una característica intrínseca de la región dado el tamaño heterogéneo de las economías que integran el APEC.

Desde su fundación, el P4 fue, por sus particulares características, el punto de partida de las nuevas configuraciones de la región asiática del Pacífico con base en subregiones con acuerdos de tipo A. Esto por diversos aspectos, entre los que destacan tres: en primer lugar, es el primer tratado de libre comercio de carácter tricontinental en la región del APEC con países de Asia, Oceanía y América Latina (Herreros, 2011:5-7); segundo, es un acuerdo de tipo A, horizontal, que vincula a cuatro economías pequeñas y abiertas que tenían bajos niveles de comercio entre sí en un acuerdo formal; y tercero, es un acuerdo abierto que permite la adhesión de nuevos países con el objetivo de promover la creación de una alianza estratégica para la liberalización del comer- 
cio en la región. Según este último punto, varios miembros del APEC ven en el P4 una plataforma fundamental para alcanzar un área de libre comercio total de sus miembros según la Declaración de Santiago de 2004.

Es relevante mencionar que, ya en el año 2008, estas características particulares llamaron la atención de Estados Unidos por la potencialidad de generar a partir del P4 un acuerdo regional mayor que amplíe la base de sus miembros, y en el que el país del norte tenga una influencia importante. En 2009 el gobierno de Barak Obama anunció que negociaría con los países del $\mathrm{P} 4$ con el objetivo antes mencionado de ampliarlo. De ese modo, comenzaron las negociaciones en marzo de 2010, lo que dio inicio al TPP. En ese marco, hasta mayo de 2012, ya se habían realizado doce rondas de negociación, con un número total de nueve participantes. En efecto, en la primera ronda, en marzo de 2010, se incorporaron Australia, Estados Unidos, Perú y Vietnam y, en la tercera ronda de ese mismo año, se incorpora Malasia, todos ellos miembros del APEC. Ha habido otros intentos de incorporación, por ejemplo, en noviembre de 2011, Japón anunció que comenzaría a consultar a los nueve miembros sobre la posibilidad de unirse a las negociaciones. El mismo año, México y Canadá manifestaron interés por sumarse a Japón en su intención de participar del TPP. Al año siguiente - en febrero - Costa Rica manifestaría la misma intención. Por último, en junio de 2012, el TPP extendería una invitación formal a México y Canadá para que se sumaran a las negociaciones del acuerdo.

Las principales características del TPP, según Herreros (2011:6), son las siguientes: primero, establece un área de libre comercio sin exclusiones de productos (excepto algunos para Brunei) e incluye capítulos sobre comercio de servicios, compras públicas, propiedad intelectual, política de competencia y solución de controversias, inversiones y servicios financieros, además de un Acuerdo de Cooperación Ambiental y un Memorando de Entendimiento sobre Cooperación Laboral. Segundo, busca generar una asociación estratégica que trascienda lo comercial, incorporando también los ámbitos económico, financiero, científico, tecnológico y de cooperación. Y tercero, tiene la meta explícita de apoyar el proceso para alcanzar el libre comercio de bienes, servicios e inversiones dentro del APEC en 2020, según lo acordado en las "Metas de Bogor". Para esto, el preámbulo del TPP afirma el compromiso de los países miembros para promover nuevas adhesiones al acuerdo - tanto de países miembros como no miembros del APEC. 
Aspecto este último relevante para América Latina, por cuanto varios países no miembros del APEC están volcando sus estrategias exportadoras al Asia. Este es el caso de Colombia - que forma parte de la Alianza del Pacífico -, de Costa Rica - que ya firmó un TLC con China -, y de países como Brasil, cuya vocación de potencia global lo mueve a mirar con expectación las alianzas que se van configurando en espacios estratégicos como la cuenca del Pacífico.

Asimismo, en esta lógica de configurar espacios estratégicos, llama la atención la no participación de China - actor clave en la región - en la conformación de este nuevo bloque TPP. En efecto, en un proceso de cooperación formal en Asia-Pacífico se esperaría que se incluyera al gigante asiático por ser la principal potencia económica del continente, y porque participa al igual que todos los miembros del TPP del foro de cooperación APEC. Uno de los motivos de esta ausencia podría ser la presencia de Estados Unidos en el TPP, lo que establece una diferencia entre la configuración de la ASEAN + 3 y el TPP en función de intereses estratégicos y de una puja entre las dos potencias por ejercer su influencia en cada bloque.

En el caso de la ASEAN + 3, se incorporaron al bloque original de diez miembros Corea del Sur, Japón y China, sin embargo, a pesar de que en el año 2009 Estados Unidos firmó un Tratado de Amistad y Cooperación (TAC) con la ASEAN (Xinhua.net 20-07-2007), y en 2010 buscó profundizar sus relaciones con este bloque en busca de elevar su vínculo a un nivel "estratégico", esto no ha tenido resultados efectivos. Por el contrario, la principal relación estratégica de la ASEAN se desarrolla con China, en especial en el ámbito económico, al ser su primero socio comercial en exportación e importación (SELA, 2010).

En consecuencia, es posible sostener que en este afán por avanzar hacia una mayor cooperación también es posible identificar espacios de competencia, con rivalidades que permean esta subregión conformada como TPP en función de los intereses de las principales potencias, Estados Unidos y China. De este modo, el interés estratégico de esta separación entre Estados Unidos y China se entiende porque la creación de un bloque exclusivamente asiático, promovido y liderado por China, dejaría a Estados Unidos en una posición económica y comercial desventajosa en la región con mayor dinamismo económico del orbe, y debilitaría incluso los fuertes vínculos políticos y estratégicos que ha mantenido con Asia Oriental desde el final de la Segunda Guerra Mun- 
dial, situación que ha generado gran preocupación en el país del norte (Herreros, 2011). Es por esto que Estados Unidos estaría apostando por la ampliación del TPP: para construir una "comunidad transpacífica" que contrarreste esta tendencia centrípeta de bloques en la zona. Lo anterior demuestra que, a pesar de los avances en la configuración de estas subregiones de libre comercio con vistas a la formación paulatina de un área total de libre comercio del APEC, hay en el proceso intereses estratégicos geopolíticos que se superponen a los principios e intereses de liberalización económica y que se reflejan en la actitud excluyente de los bloques integrados por cada una de las dos grandes potencias de la región, Estados Unidos y China.

\section{CHINA Y LA ASEAN EN LAS NUEVAS CONFIGURACIONES DEL ASIA-PACÍFICO}

Una de las experiencias de integración regional más antiguas en nuestro análisis es la ASEAN, organización fundada en 1967 por cinco países, Malasia, Indonesia, Tailandia, Singapur y Filipinas, y que desde 1989 es parte del APEC. Aunque los orígenes de la ASEAN sean muy distintos a los principios del APEC dada su motivación política, el contexto de inicio de los noventa llevará a esta subregión a ser un actor muy importante para el dinamismo económico de la región asiática del Pacífico, principalmente desde que los diez países miembros firmaron un tratado de libre comercio el año $1992^{3}$.

En un principio, la ASEAN se planteó como una iniciativa de cooperación regional política declarando una zona de paz que permitiera un contexto regional libre de conflictos para que estos Estados avanzaran en sus procesos de consolidación e integración nacional. Recordemos que, a excepción de Indonesia, los cuatro países miembros fueron colonias hasta la década del cincuenta.

Sobre esa base de intereses comunes, hay otras dos características que podemos mencionar (Severino, 2007): primero, la voluntad de estas cinco naciones no comunistas y con economía de mercado del Sudeste asiático de evitar la expansión del comunismo de Vietnam hacia los países vecinos; $y$, segundo, la necesidad de asegurar el uso de métodos pacíficos para la resolución de los múltiples conflictos territoriales - algunos todavía vigentes - de la subregión, buscando la paz y estabilidad regional. Esto último se reflejó en la firma - en el mismo año de la fundación de la organización - del Tratado de Amistad y Cooperación 
del Sureste Asiático (Tacse) que los declara una zona de neutralidad ante el conflicto de la Guerra Fría y la influencia de las dos grandes potencias: Estados Unidos y la Unión Soviética.

Sin embargo, con el pasar de los años, la ASEAN se irá transformando en un proceso exitoso de integración regional al que se incorporarán Brunei, en 1984, Vietnam, en 1995 - hecho de suma relevancia considerando que la organización se funda en oposición a ellos mismos-, Laos y Myanmar, en 1997, y Camboya, en 1999. Esta transformación se desarrollará principalmente en el periodo post Guerra Fría, en un nuevo contexto internacional, y tras años de crecimiento económico sostenido en la región - sobre todo en los llamados "Cuatro Tigres Asiáticos" (Taiwán, Corea del Sur, Hong Kong y el miembro de la ASEAN, Singapur), dinamismo que refuerza China desde las reformas económicas de apertura en 1978. Por lo tanto, será a fines de la década de 1990, en la sexta cumbre de la ASEAN en 1998, que se acordará establecer contacto de forma regular con la República de Corea, Japón y la República Popular China, en lo que se denomina ASEAN + 3, paso importante para la organización al vincularse con las principales potencias económicas de la región ${ }^{4}$.

Analicemos a continuación el rol de China al firmar un tratado de libre comercio con la ASEAN. En efecto, desde la década de 1990, China ha mostrado un interés activo en la cooperación regional principalmente desde los puntos de vista político y militar. Durante la Guerra Fría, especialmente hasta la década de 1970, la relación de China con muchos países del Sudeste Asiático se había deteriorado. Desde la década de 1990, en cambio, China trabajó activamente para mejorar las relaciones políticas y comerciales con los países del Sudeste Asiático, mejorando las relaciones con Indonesia y Vietnam, y estableciendo relaciones diplomáticas con Singapur.

Sin embargo, desde la crisis financiera asiática en el año 1997, la importancia de la cooperación económica en sus relaciones con la ASEAN se ha incrementado con el propósito de formalizar acuerdos económicos. Zhu Rongji, primer ministro chino en ese entonces, propuso a la ASEAN la creación de un tratado de libre comercio "dentro de los próximos diez años", en noviembre de 2000. El primero de estos acuerdos nace de la sexta cumbre China-ASEAN realizada en noviembre de 2002. En dicha reunión, los representantes de la ASEAN y el gigante asiático firman el "Acuerdo Marco sobre Cooperación Económica Glo- 
bal China-ASEAN", punto de partida en este proceso de acercamiento e intensificación de intercambio entre ambos. Dos años más tarde, se firmará el "Acuerdo sobre Comercio de Mercancías" dentro del TLC, que entrará en vigor en 2005, y en 2007 y 2009 se firmarán los acuerdos sobre comercio de servicios y el relativo a inversiones, respectivamente. Todos ellos se establecen dentro de este acuerdo de libre comercio que, el primero de enero de 2010, entra en vigor como Zona de Libre Comercio con el objetivo de reducir los aranceles entre sus miembros en un 90 por ciento, en lo que será el mayor acuerdo de libre comercio del orbe por número de consumidores - con casi 1,9 billones de personas -, e incorporará a parte de las economías líderes en exportación a nivel mundial (BBC News, 24/10/2011).

De alguna manera, China quiso tranquilizar a los países de la ASEANque se sentían amenazados por su crecimiento económico - dando mayor importancia a las relaciones políticas y estratégicas con estos países por encima de lo económico. Sin embargo, este acuerdo de China con la ASEAN dio un estímulo considerable a Corea del Sur y Japón que hasta ese momento habían tenido una actitud pasiva en la formalización de tratados de libre comercio en Asia - pero que sí lo hacían con países latinoamericanos -, lo que motivó el movimiento hacia la integración económica de la ASEAN+3 con base en un acuerdo tipo A.

Así, en octubre de 2003, los gobiernos de Japón y los diez países miembros de la ASEAN firman el Framework Agreement for Comprehensive Economic Partnership o Acuerdo Marco Japón-ASEAN (Bilaterals.org, $21 / 11 / 2007$ ) y, en noviembre de 2004, se inician las negociaciones. Esto responde al interés de Japón de participar en esta reestructuración regional, al ver los avances existentes entre China y la ASEAN y las negociaciones en paralelo entre la ASEAN y Corea del Sur. Luego de once rondas de negociación, se acuerda la firma del TLC en noviembre de 2007, que entra en vigor en 2008. Este acuerdo es de amplio alcance e incluye el comercio de bienes, servicios, inversión y cooperación económica. De esa forma, el comercio total entre la ASEAN y Japón se expande en un 22,5 por ciento - al pasar de 173,1 mil millones de dólares en 2007 a 214,4 mil millones de dólares en 2008 - y, a pesar de verse afectado por la crisis, sigue siendo importante para ambos hasta la actualidad (Bilaterals.org, 21-11-2007).

Asimismo, Corea del Sur negocia en este mismo periodo con la ASEAN con el objetivo de concretar un TLC, propuesta hecha por el presidente 
coreano Roh Moo Hyun en octubre de 2003. Dos años después, fue firmado en el marco de la Cumbre ASEAN - Corea del Sur el "Acuerdo Marco sobre Cooperación Económica Global" entre ambas partes. En 2007, se concreta el "Acuerdo sobre el Comercio de Servicios" firmado por nueve miembros de la ASEAN y Corea del Sur, y finalmente, el primero de enero de 2010, entra en vigor el Área de Libre Comercio ASEAN - Corea del Sur, tras años de negociaciones y con cierta flexibilidad con algunos miembros de la ASEAN ${ }^{5}$ (Bilaterals.org, 2/6/2009). Las relaciones comerciales entre ambos se mantuvieron sólidas incluso en el periodo de crisis económica y financiera mundial, aunque su comercio total descendió levemente en 2009 (4,5 por ciento) respecto del año anterior, y alcanzó el nivel de 74,7 mil millones de dólares a 78,3 mil millones en 2008. El comercio entre ambos casi se triplicó entre los años 2001 y 2008 - pasó de 32 mil millones de dólares a 90 mil millones (Bilaterals.org, 2/6/2009).

En consecuencia, este proceso de la ASEAN de formalizar acuerdos tipo A en la región es parte también de la nueva configuración de subregiones del APEC. Los tratados firmados entre la ASEAN y los tres países que componen el bloque " +3 ", se negocian en los primeros años del siglo XXI y se consolidan a partir de 2004, momento en que comienzan a proliferar este tipo de acuerdos en el marco del APEC - tanto China como Corea del Sur y Japón son miembros de este foro, además de todos los miembros de la ASEAN. Es importante también mencionar que, en febrero de 2009, la ASEAN firmó un tratado de libre comercio con Australia y Nueva Zelanda (ambos miembros de APEC), que entró en vigor en 2010 para casi todos los miembros de la ASEAN, además de otro, en agosto del mismo año, con India, país que, hace más de una década, intentó ingresar en este foro económico (Karl, 2011). Lo anterior muestra, al igual que en el caso del TPP, que la nueva configuración del APEC en base a subregiones no es excluyente, porque a través de los TLC entre los miembros de la APEC y los no miembros será posible también considerar un área de libre comercio del Asia-Pacífico de más de veintiuna economías.

Asimismo, en esta lógica de configurar espacios estratégicos, llama la atención la ausencia de Estados Unidos como actor clave en la conformación de esta subregión ASEAN+3. En general, Estados Unidos ha presentado una actitud de oposición a la integración regional de ASEAN+3, ya que ésta lo excluye. Estados Unidos manifestó claramente posiciones opuestas, por ejemplo, a la creación del Fondo Mone- 
tario Asiático que Japón había propuesto después de la crisis financiera de Asia oriental en 1997. Como alternativa, entonces, Estados Unidos ha colocado sus esfuerzos en aprovechar el espacio del APEC con el fin de consolidar el TPP para generar equilibrio con ASEAN+3.

En estas circunstancias, los TLC entre los países de Asia oriental progresan rápidamente y Estados Unidos también comenzó a prestar atención a un tratado con países de esta región. Desde el año 2002, Estados Unidos comenzó a mostrar un mayor interés y voluntad de avanzar en la negociación con los países de la ASEAN, cuyo resultado ha sido la firma de un tratado de libre comercio con Singapur en el año 2003 y con Corea del Sur en el año 2007 (Xinhua.net, 20/7/2007). Es por esto que Estados Unidos se centrará en fortalecer la cooperación bilateral con los países de Asia oriental, pero también juega liderando una subregión, y se esfuerza por la concreción del TPP que excluye, hasta ahora, a China.

\section{AMÉRICA LATINA EN LAS NUEVAS CONFIGURACIONES DEL ASIA-PACÍFICO: CHILE, PERÚ, MÉXICO Y COLOMBIA}

Uno de los proyectos más recientes de integración regional en América Latina y el Caribe (ALC), es la AP, acuerdo firmado en junio de 2012 entre Chile, México, Colombia y Perú - con Panamá y Costa Rica como observadores. Es un proyecto que se podría comparar en cuanto a magnitud sólo con el Mercosur a nivel regional - aunque tendría un comercio total mayor ${ }^{6}-$, y que busca generar un nuevo bloque de integración profunda que vaya más allá de un tratado de libre comercio, es decir, un acuerdo tipo $\mathbf{B}$, que se propone gestionar mayores beneficios para sus miembros al ser una plataforma conjunta que les permita negociar en mejor pie con Asia.

El hecho de que la AP sea un acuerdo tipo B es novedoso por dos razones que queremos explicar en las siguientes líneas. Primero, porque el regionalismo abierto que se inicia en los años noventa en América Latina es considerado en la actualidad por diversos autores como una integración estancada o en crisis (Malamud, 2009; Gudynas, 2005; García, 2010; Borbón, 2007; Bywaters y Rodríguez et al., 2009), por lo tanto, aun cuando este nuevo acuerdo apunta a formar un mercado común, esto debiera ser algo diferente al regionalismo latinoamericano tradicionalmente abierto. Y segundo, en la AP están dos países, Chile y México, 
que hasta ahora no habían participado en ningún bloque del regionalismo abierto latinoamericano.

Más ampliamente, lo que diferencia este acuerdo de la AP del regionalismo abierto tradicional es que busca formar un mercado común para aumentar el comercio con Asia y apoyarse para generar una industria local que les permita ingresar en cadenas productivas (Direcon, $29 / 4 / 2011$ ). Esto difiere del regionalismo de los años 1990, en el cual los países buscaban aumentar el comercio intrabloque, objetivo que tenía importantes limitaciones ya que la mayoría de los países latinoamericanos exportaban fuera de la región, principalmente a Estados Unidos y Europa. En consecuencia, la Alianza del Pacífico sigue el modelo de acuerdos tipo B, pero es de nuevo tipo ya que responde a las nuevas configuraciones económicas del Asia Pacífico, considerando que su creación y objetivos están puestos en el comercio con Asia. Esto explica que participen Chile y México, que son países con una inserción económica internacional basada, hasta el momento, sólo en tratados de libre comercio, pero que ahora firmaron un acuerdo tipo B porque los objetivos de la AP coinciden con sus intereses por el mercado asiático.

Revisemos algunos antecedentes de la creación de este acuerdo para identificar qué es lo nuevo de esta participación latinoamericana en las nuevas configuraciones económicas del Asia Pacífico.

Al respecto, debemos señalar que es en el Foro Arco del Pacífico - que surge el año 2006 y se consolida el 2008 - donde surgirá la propuesta para conformar la AP, que se define como "un espacio informal de coordinación y concertación de alto nivel para la identificación e implementación de acciones conjuntas dirigidas a generar sinergias entre los países participantes en materia económica y comercial, teniendo en cuenta su interés común de fortalecer sus relaciones con las economías del Asia Pacífico" (Arco del Pacífico.org, 31/10/2008). Sus temas son principalmente económicos y comerciales: fomentar las inversiones, cooperación técnica y facilitar el comercio entre sus once países miembros: Chile, Colombia, Costa Rica, Ecuador, El Salvador, Guatemala, Honduras, México, Nicaragua, Panamá y Perú.

El Arco del Pacífico fue una iniciativa promovida por el ex presidente peruano Alan García (2006-2011) y respondió a un contexto regional complejo, dados los cambios que comenzó a experimentar la región a inicios del siglo XXI. Entre ellos el ascenso, a principios de siglo, de go- 
biernos de izquierda en América Latina (Correa en Ecuador, Morales en Bolivia, Ortega en Nicaragua), críticos de los modelos de regionalismo abierto y de la proliferación de tratados de libre comercio, con propuestas de modelos de integración posliberal como el ALBA y Unasur (Briceño, 2010). Más concretamente, el año 2006, Hugo Chávez, Presidente de Venezuela, anunció su decisión de sacar a su país de la CAN, lo que generó una crisis en dicha organización, que ya venía siendo cuestionada en sus metas de integración por el incumplimiento de algunos de los reglamentos por sus países miembros. Ante esto, García buscó promover un mecanismo de cooperación paralelo a la CAN incluyendo a Chile - que integrara a los países ribereños del Pacífico (Briceño, 2010) ${ }^{7}$. En una primera instancia, se pensó crear un bloque dentro del APEC, pero luego se modificó la estrategia para incluir a todos los países que dan a la ribera del Pacífico, fuesen o no de este foro, considerando que los países latinoamericanos que están geográficamente en la Cuenca del Pacífico son más que Perú, Chile y México, los únicos países miembros de APEC.

Por lo tanto, el Arco del Pacífico cumpliría tres funciones (Briceño, 2010:55): 1) como bastión para defender las políticas neoliberales aplicadas desde los años 1980; 2) como una respuesta al ALBA y su propuesta de crear un "eje antisistémico", no capitalista y anti-imperialista; y 3) como instancia para tratar de institucionalizar un espacio regional a partir del cual se pueda negociar de mejor forma con Asia, especialmente con China.

Respecto de la última función, es interesante remarcar que el comercio con Asia ha sido, desde principios de siglo, muy importante para América Latina. Chile destinó para el 2010 el 34 por ciento de sus exportaciones a esa región, Perú un 22 por ciento y Costa Rica un 12 por ciento (Briceño, 2010:57), además, estos tres países tienen tratados de libre comercio con China, los únicos en la región. En consecuencia, el Arco del Pacífico se constituyó como un espacio de cooperación, negociación y generación de confianza entre los países miembros de la Cuenca del Pacífico, propicio para el nacimiento de un nuevo proyecto intrarregional, la AP.

La AP comenzó a ser insinuada por el ex primer mandatario peruano, a mediados de 2010, en una carta escrita en octubre de dicho año a sus pares de Chile, Perú, Colombia y Panamá para invitarlos a participar de ella. Sin embargo, no será hasta la Cumbre Iberoamericana en Mar 
del Plata en diciembre de 2010 - tras la invitación en octubre del presidente chileno Sebastián Piñera a una reunión para discutir la iniciativa - que toma forma la idea de concretar un acuerdo de integración profunda entre ellos.

Tras estas reuniones - sumada a una ministerial a principios de 2011 se firmó el 29 de abril de 2012 en Lima, Perú, la "Declaración Presidencial sobre la Alianza del Pacífico", en donde se establecieron las motivaciones de dicha iniciativa, sus objetivos y posiciones sobre la integración regional y las relaciones con el resto del mundo, especialmente con Asia, aspectos que serán plasmados en el Acuerdo de la AP firmado en junio de 2012. En este acuerdo, se señala que lo que se busca es "conformar un Área de Integración Profunda mediante un proceso de articulación política, económica y de cooperación e integración en América Latina" (Direcon, 29/4/2011). Al respecto, llama la atención la utilización de dos conceptos. Primero, el de "integración profunda", que implica un proceso de integración regional multidimensional - no sólo de cooperación económica, sino también social, política, cultural, energética, etc. - idealmente con tintes de supranacionalidad, para la adopción y aplicación de políticas comunes en esas diversas dimensiones. Algunos logros en esta línea, en junio de 2013, son el libre paso de personas entre los cuatro países miembros y el intercambio estudiantil. Asimismo, se espera que en el futuro las personas puedan trabajar libremente en cualquiera de las naciones que integran el grupo, al tiempo que su seguridad social (previsión) también se pueda mover con ellos. Otro avance en esta línea es la conformación de una representación extranjera común, siendo en Ghana la primera embajada con estas características (La Tercera, 2013).

Y, segundo, el concepto de "América Latina", lo que no es azaroso sino deliberado puesto que implica la inclusión principalmente de México y los países del Caribe, no considerados en otros procesos de integración regional - como el Mercosur o Unasur, en donde se utiliza el concepto de Suramérica, o América del Sur. A ello hay que agregar que la Alianza, en junio de 2013, ha aceptado la integración de Costa Rica, una vez que ratifique el Tratado de Libre Comercio con Colombia, así como el Acuerdo Marco de la Alianza, que se espera lo haga a finales de este mismo año.

Debemos agregar también que en la Declaración (Direcon, 29/4/2011) se desarrolla un aspecto clave, según el cual se ratifica el compromiso 
de los países miembros "de privilegiar la integración como un instrumento efectivo para mejorar el desarrollo económico y social de nuestros pueblos, promover las capacidades de emprendimiento y avanzar hacia un espacio amplio que resulte más atractivo para las inversiones y el comercio de bienes y servicios, de manera que proyecte a nuestros países con mayor competitividad, especialmente hacia la región del Asia-Pacífico". Esto muestra la visión de los países firmantes respecto de lo que consideran la integración regional: un instrumento para el desarrollo económico y social de cada Estado partícipe y del bloque en su conjunto en la medida que les permite alcanzar un grado mayor de competitividad, especialmente hacia el continente asiático, además de señalar que los tratados de libre comercio ofrecen una plataforma que facilita y propicia la integración de sus economías.

Por último, antes de pasar a sus objetivos, se afirma que "los acuerdos que alcancemos en el marco de esta iniciativa deberán contribuir y profundizar los acuerdos económicos, comerciales y de integración que nuestros países hayan suscrito a nivel bilateral, regional y multilateral" (Direcon, 29/4/2011). Esto es interesante de considerar, puesto que una de las características de los miembros es la preexistencia de acuerdos entre ellos, que en vez de eliminarse por esta nueva iniciativa podrá profundizarse y homologarse, algo positivo en términos prácticos para la integración latinoamericana, considerando lo que la Asociación Latinoamericana de Integración (Aladi) denomina el "bilateralismo convergente", que se explicará posteriormente.

Este acuerdo tipo B parece incorporar elementos que efectivamente podrían reflejar un grado de integración profunda, tales como la libre circulación de personas, la cooperación policial y la interconexión energética y física, además de plantear la posible creación de algún tipo de mecanismo de solución de diferencias.

La AP entonces es una iniciativa novedosa e interesante por tres motivos. Primero, incluye a cuatro países - sin considerar a dos en calidad de observador - de la Cuenca del Pacífico que comparten características comunes, como su situación geográfica, su estabilidad económica y alta inversión extranjera, su apego al regionalismo abierto, su cercanía con Estados Unidos y la Unión Europea, y sobre todo, el hecho de que comparten los tratados de libre comercio entre ellos - salvo México y Perú, pero está en negociación - y ven en la región Asia-Pacífico un potencial comercial importante. 
En segundo lugar, y esto es más una interrogante que algo establecido, para que efectivamente sea un proyecto de integración "profunda", la AP deberá incluir una institucionalidad supranacional independiente de los Estados miembros - con personalidad jurídica - que sea capaz de generar, coordinar y velar por el cumplimiento de políticas y normas comunes para alcanzar los objetivos establecidos en la primera etapa del acuerdo: "movimiento de personas, de negocios y facilitación para el tránsito migratorio, incluyendo la cooperación policial; comercio e integración; facilitación de comercio y cooperación aduanera; servicios y capitales, incluyendo la posibilidad de integrar las bolsas de valores y mecanismos de solución de diferencias" (Nuñez, 5/7/2012).

Y, en tercer lugar, lo interesante de este bloque con relación al vínculo Asia-Pacífico es que podrá, en la medida que se consolide internamente y sea capaz de coordinar sus políticas comerciales, negociar en mejor pie con el continente asiático, puesto que en la actualidad China negocia bilateralmente con la mayoría de los miembros de la AP en una relación asimétrica que la beneficia enormemente. Por lo tanto, si tuviese que negociar con un bloque, la asimetría podría ser menor. Algunos datos refuerzan esta idea: Chile, Perú, México y Colombia representan el 40 por ciento del Producto Geográfico Bruto de América Latina y el 55 por ciento de todas sus exportaciones, además de que juntos estos países cuentan con cerca de 206 millones de consumidores, con un PIB conjunto de cerca de 1,7 billones de dólares (Nuñez, 5/7/2012).

En conclusión, la AP considera aspectos que van más allá de la mera cooperación económica, por lo que podríamos presenciar una organización de integración regional distinta a las anteriores en América Latina. Se ha incluido además a dos países que suelen no estar en los procesos de integración regional, Chile y México, aspecto positivo, puesto que cubre una parte del continente que no ha avanzado en este tema. De esta manera, esta subregión ubicada en el continente americano se suma a las nuevas configuraciones que hemos analizado del Asia-Pacífico, pues la AP nace y se proyecta por el comercio con el Asia. Sin embargo, la AP aporta un nuevo modelo a estas nuevas configuraciones ya que no sólo es un tratado de libre comercio sino que es un proyecto para una integración profunda, que en este análisis hemos denominado acuerdos de tipo B, llevando así el regionalismo formal a las dinámicas de cooperación y concertación del APEC. 
Chile en lo específico, desarrolló un regionalismo abierto, flexible y dinámico, que lo privó hasta hora de participar como miembro pleno de algún bloque subregional en América Latina, y compensa lo anterior con una estrategia ofensiva, en un sentido activo y dinámico, de apertura comercial hacia distintos países y regiones. Chile tiene acuerdos de complementación económica, acuerdos de asociación económica y acuerdos de libre comercio con la mayoría de los países de América Latina, con países desarrollados como Canadá, Estados Unidos y la Unión Europea, y, además, con importantes economías del Asia, como China, India, Japón y Corea del Sur, entre otros, sumando más de veintiséis acuerdos en total. No obstante, al igual que México, que suma también más de veinte acuerdos en total, ahora estrena una nueva estrategia de relación con Asia, bajo un regionalismo abierto formal de nuevo tipo, que focaliza su proyección y potencial en el mercado asiático.

Con relación a las dos grandes economías de esta nueva configuración económica del Asia-Pacífico, Estados Unidos y China, debemos señalar que ambos están fuera de la AP. Claramente, China es un móvil del acuerdo de la AP: cuando estos cuatro países se plantean aumentar el comercio con Asia, gran parte de ello está concentrado en el comercio con China. Hay en este nuevo bloque subregional una oportunidad para enfrentar en mejor pie a China y al continente asiático, economías dinámicas que proyectan mantener su crecimiento a futuro y que, si bien proporcionan buenas ganancias a los países latinoamericanos, podrían producir ganancias aún mayores si estos países negociaran desde una posición menos asimétrica. Sería interesante ver a Chile y Perú negociando conjuntamente las condiciones de venta hacia China del cobre, recurso natural que el gigante asiático demanda y seguirá demandando al menos en los próximos veinte años para mantener su ritmo de crecimiento.

Con respecto a Estados Unidos, la gran interrogante es su presencia y la posibilidad de que efectivamente intente una especie de Área de Libre Comercio de las Américas (ALCA) en una versión actualizada. Hay que recordar que los cuatro países miembros de la AP han firmado tratados de libre comercio con Estados Unidos, es decir, son miembros del ALCA, proyecto que intentó incluir a toda la región el año 2005. De todas formas, la AP surge en el marco del Arco del Pacífico y tiene como uno de sus objetivos principales el generar un bloque que coordine sus políticas comerciales para relacionarse con el continente asiáti- 
co, por lo que no debería incluir a Estados Unidos y sí concentrarse en la región. Según el análisis de Uziel Nogueira, la AP tiene repercusiones en la geopolítica regional, considerando que México garantiza su presencia política en Sudamérica y con respecto a Brasil, por lo que a partir de ahora tendrá necesariamente que agregar a México en su estrategia de integración sudamericana. A su vez, Estados Unidos refuerza su presencia en Sudamérica vía México (Nogueira, 19/6/2012), lo que afecta el equilibrio bipolar de poder entre Brasil y Venezuela. Adicionalmente, la AP es un nuevo instrumento para apoyar a dos principales "aliados" de Estados Unidos: Chile por los aspectos comerciales y Colombia por lo militar y estratégico. Lo último con repercusión en la percepción de Venezuela de la AP, la cual es bastante crítica por la relación y afinidad de estos países con Estados Unidos en el plano político y estratégico. Por su parte, Brasil concentra su crítica en que la AP no es un proceso de integración profunda como sí lo es el Mercosur, que cuenta, además, con la membresía de las dos potencias regionales Brasil y Venezuela.

\section{CONCLUSIONES}

Este artículo ha analizado las nuevas dinámicas de cooperación económica que se dan a partir del año 2004 en la región del Asia-Pacífico tomando como núcleo central el APEC, que, desde 1989, impulsa la liberalización unilateral concertada de veintiuna economías que dan a la Cuenca del Pacífico. Estas nuevas configuraciones de relaciones económicas se han formalizado con la firma de tratados de libre comercio creando subregiones estratégicas que se mueven en dos dinámicas: por un lado, una dinámica de cooperación que busca avanzar en función de la meta del APEC 2020, que consiste en formar un área de libre comercio regional en la Cuenca del Pacífico, y por otro lado, dinámicas de rivalidad económica, política y estratégica que permean estas subregiones en función de los intereses de las principales potencias de la región, Estados Unidos y China.

Al respecto, constatamos en el análisis que estas nuevas configuraciones económicas en la región del Asia-Pacífico desde el 2004 surgen de la Cumbre del APEC en Santiago de Chile, en la cual las veintiuna economías miembros reconocen la firma de tratados de libre comercio de alcance parcial entre ellas como una vía aceptable para alcanzar la meta de formar un área de libre comercio total en la región en 2020. Con 
ello, el APEC pasa de un regionalismo informal y concertado a un regionalismo formal con la firma de TLC.

Asimismo, es posible comprobar la existencia de estas configuraciones económicas como resultado de este proceso iniciado el 2004, por medio del análisis del surgimiento de las subregiones económicas, que se inician con la creación del P4 y que siguen con ASEAN+3, el TPP y - la más reciente - la AP. Las características de estas subregiones es que se forman con base en acuerdos de libre comercio que, según la conceptualización de Di Filippo y Franco (2000), hemos llamado de tipo A para diferenciarlas de los acuerdos de tipo B que serían acuerdos de integración que van más allá de un TLC.

No obstante, al analizar la participación de los países de América Latina miembros del APEC - Perú, Chile y México - que conforman la subregión de la AP (que incluye a Colombia y se espera que en 2014 incluya a Costa Rica) se observa que este acuerdo no sólo es un área de libre comercio, sino que anida un proyecto de integración profunda, un acuerdo tipo B, que incluye temas como la interconexión física y energética, la cooperación policial, y la libre circulación de bienes y servicios, así como también de personas.

La afirmación de que la AP es más que un tratado de libre comercio se fundamenta en que estos cuatro países ya tienen tratados de libre comercio firmados entre sí. En consecuencia, la estrategia de esta subregión de América Latina es insertarse en el Asia-Pacífico con una integración de nuevo tipo, tipo B, con vistas a conformar un mercado común para potenciar de la mejor forma sus recursos en las negociaciones con el mercado asiático e impulsar y apoyar los intereses comunes por desarrollar la industria local. Hasta junio de 2013, los países de la AP han avanzado en el libre ingreso de personas a sus respectivos Estados y en la mejora de las condiciones de intercambio estudiantil, así como también han gestado una representación diplomática común que buscan implementar desde finales del mismo año.

Lo interesante es que el proyecto de la AP también resulta novedoso en el contexto latinoamericano en comparación con bloques como el Mercosur y la CAN, entre otros, puesto que la AP incluye a Chile y México, países que no habían participado hasta ahora de acuerdos tipo B. Chile se había destacado por firmar sólo acuerdos tipo A, con 26 acuerdos firmados (17 TLC, 4 Acuerdos de Asociación Económica y 6 Acuerdos de Complementación Económica) hasta la fecha de este artículo, y Méxi- 
co, por su parte, suma 10 TLC, 30 Acuerdos de Promoción y Protección de Inversiones, más 9 Acuerdos de Complementación Económica (Secretaría de Economía, 2013; Direcon, 2013).

Con respecto a las dinámicas de rivalidad económica, política y estratégica que permean estas subregiones en función de los intereses de las principales potencias de la región de Asia-Pacífico, como Estados Unidos y China, es posible sostener algunas conclusiones generales y parciales. El análisis muestra que Estados Unidos juega con ganar influencia al ser parte del acuerdo TPP (a la fecha de este artículo todavía en negociación) y China busca reforzar su liderazgo con ASEAN+3. Ambas potencias proyectan en estas subregiones sus intereses económicos pero permeados por sus intereses estratégicos y geopolíticos derivados de su condición de potencias. También Estados Unidos podrá tangencialmente tener influencia en América Latina a través de su cercanía con los tratados de libre comercio con los países miembros de la AP, Perú, Chile, México y Colombia. Queda abierta la interrogante sobre su futura influencia en la región, por ejemplo, si intentará desplazar la influencia de Brasil posicionando de mejor forma a México como actor de una integración de nuevo tipo que se plantea de cara a los desafíos que presenta el mercado asiático. Lo mismo para Venezuela, que lidera un bloque de integración como el ALBA y participa junto a Brasil en Mercosur.

(Recebido para publicação em dezembro de 2012)

(Reapresentado em setembro de 2013)

(Aprovado para publicação em outubro de 2013) 


\section{Isabel Rodríguez Aranda}

\section{NOTAS}

1. Las metas de Bogor, que abogan por una mayor liberalización y apertura en el comercio y la inversión, fueron establecidas por APEC en su reunión de 1994 en Bogor (Indonesia).

2. La Asociación de Naciones del Sudeste Asiático - ANSA (en inglés: Association of Southeast Asian Nations - ASEAN) es una organización regional de estados del sudeste asiático creada el 8 de agosto de 1967.

3. Hoy, la ASEAN está integrada por Indonesia, Malasia, Filipinas, Singapur, Tailandia, Brunei, Vietnam, Laos, Birmania y Camboya.

4. Vinculación que es principalmente económica ya que la ASEAN tiene también una arista de cooperación en seguridad regional a través del Foro de Cooperación de la ASEAN (ARF, por su sigla en inglés).

5. Para Brunei Darussalam, Indonesia, Malasia, Filipinas, Singapur y Tailandia, hasta 2012; con Vietnam, hasta 2016; y con Camboya, la República Democrática Popular Laos y Myanmar, hasta 2018.

6. En palabras del Presidente mexicano Felipe Calderón: "Mientras el comercio total del Mercosur llega a US\$543.000 millones, la Alianza del Pacífico tendrá 872.000 millones" (La Nación, 15/5/2011).

7. En palabras del canciller peruano José Antonio García Belaunde "La Alianza del Pacífico sí puede constituirse en la vanguardia del arco del Pacífico y ser una alternativa más ambiciosa que la CAN" (La Nación, 15/5/2011). 


\section{REFERENCIAS}

ARCO DEL PACÍFICO.ORG. (2008), “Declaración de los Jefes de Estado de los Países Miembros del Foro del Arco del Pacífico Latinoamericano". El Salvador, 31 de octubre (capturado el 9 de mayo de 2012 en http://www.arcodelpacifico.org/nosotros/ cumbres).

ARMANET Pilar, ALAMOS Pilar, O’SHEA Luz. (1996), Las Relaciones de Chile con los Organismos Multilaterales de la Cuenca del Pacífico. Santiago de Chile, Instituto de Estudios Internacionales, Universidad de Chile y Fundación Chilena del Pacífico.

BBC NEWS. (2011), “China's Uneasy Relationship with Business Neighbours”, 24 de octubre (capturado el 1 de abril de 2012 en http://www.bbc.co.uk/news/business15420935).

BIBLIOTECA DEL CONGRESO NACIONAL. (2004), “Declaración de Santiago. Una Comunidad, Nuestro Futuro", 21 de noviembre (capturado el 5 de junio de 2012 en http:/ / www.bcn.cl/carpeta_temas/temas_portada.2005-10-27.7993379546/folder. 2005-10-31.1708606454/DeclaracionFinalAELM.pdf).

BILATERALS.ORG. (2007), “Japón y ASEAN Sellan un TLC", 21 de noviembre (capturado el 10 de abril de 2012 en http:/ / www.bilaterals.org/spip.php?article10413).

. (2009), “Corea del Sur y ASEAN Firman TLC para Inversión”, 2 Junio (capturado el 5 de abril de 2012 en http:/ / www.bilaterals.org/spip.php?article15201).

BORBÓN, Josette Altmann. (2006), “Integración Latinoamericana: Crónica de una Crisis Anunciada". OBREAL/ EULARO, no 33, 15 de septiembre, pp. 1-16.

BRICEÑO, José. (2010), “La Iniciativa del Arco del Pacífico Latinoamericano: Un Nuevo Actor en el Escenario de la Integración Regional". Revista Nueva Sociedad, no 228, pp. 44-59.

BYWATERS, Rodríguez et al. (2009), “UNASUR y la Integración Latinoamericana: Propuesta de un Nuevo Modelo del Regionalismo Postliberal". Revista Encrucijada Americana, año 3, no 1, pp. 4-26.

DIRECOM. (2011), “Declaración Presidencial sobre la Alianza del Pacífico", 29 de abril (capturado el 20 de abril de 2012 en http:/ / www.direcon.gob.cl/noticia/3066).

. (2013), "Cuadro Resumen de Acuerdo" (capturado el 15 de agosto de 2013 en http:/ / www.direcon.gob.cl/pagina/1897).

DI FILIPPO, Armando y FRANCO, Rolando. (2000), Integración Regional, Desarrollo y Equidad. México, Editorial Siglo XXI.

GARCÍA, Carlos Alberto Chaves. (2010), “Tendencias de la Inserción Internacional de Suramérica en el siglo XXI". Revista de Ciencias Sociales, no 38, pp. 29-40.

GARCÍA, Caterina. (2006), “El Regionalismo en Asia Oriental”, en M. Spoor y S. Golden (eds.), Regionalismo y Desarrollo en Asia. Procesos, Modelos y Tendencias. Barcelona, CIDOB.

GUDYNAS, Eduardo. (2005), “El 'Regionalismo Abierto' de la CEPAL: Insuficiente y Confuso". Observatorio Hemisférico, 28 de septiembre, pp. 1-4. 
HERREROS, Sebastián. (2011), “El Acuerdo Estratégico Transpacífico de Asociación Económica: Una Perspectiva Latinoamericana". Puentes, vol. 13, no 3, pp. 5-7.

IBAÑEZ, Josep. (2000), “El Nuevo Regionalismo Latinoamericano en los Años Noventa". Revista Electrónica de Estudios Internacionales, no 1, pp. 1-11.

KARL, David J. (2011), “Knocking on APEC's Door”. Foreign Policy Association, 21 de septiembre de 2001 (capturado el 10 de abril de 2012 en http:// foreignpolicyblogs. com/2011/09/21/knocking-on-apec\%E2\%80\%99s-door/).

LA NACIÓN. (2011), “Alianza del Pacífico Clausuró su Cumbre con Declaración de Mérida", 5 de junio (capturado el 20 de mayo de 2012 en http: / / www.lanacion.cl/alianza-de-pacifico-clausuro-su-cumbre-con-declaracion-de-merida/noticias / 2011-12-05/073155.html).

LA TERCERA. (2013), "Alianza del Pacífico Logra Avances Clave en Libre Circulación de Personas y Bienes a un Año de Creación", 8 de junio (capturado el 15 de agosto de 2013 en http://www.latercera.com/noticia/mundo/2013/06/678-527255-9-alianza-del-pacifico-logra-avances-clave-en-libre-circulacion-de-personas-y.shtml).

LÓPEZ AYMÉS, Juan. (2002), “APEC: Orígenes, Evolución, Organización, Estructura y Relevancia". El Mercado de Valores, no 15, pp. 12-23.

MALAMUD, Carlos. (2009), “La Crisis de la Integración se Juega en Casa”. Revista Nueva Sociedad, no 219, pp. 97-112.

NOGUEIRA, Uziel. (2012), “Alianza del Pacifico: ¿Nuevo Proceso de Integración o Extensión del ALCA?. Dossier Geopolítico, 19 de junio (capturado el 25 de junio de 2012 en http://www.dossiergeopolitico.com/2012/06/alianza-del-pacifico-\% C2\%BFnuevo-proceso-de-integracion-o-extension-del-alca.html).

NUÑEZ, Rogelio. (2012), "La Alianza del Pacífico, el Nacimiento de un Coloso". INFOLATAM, 5 de julio (capturado el 20 de junio de 2012 en http://www.opeal. net $/$ index.php?option $=$ com_k2\&view $=$ item\&id $=11255: 1 a-$ alianza-del- pac $\%$ C3\%ADfico-el-nacimiento-de-un-coloso\&Itemid=123).

RODRÍGUEZ, Isabel. (2005), “APEC 2004: Desde la Regionalización al Regionalismo”. Revista Diplomacia, no 102, pp. 68-82.

SANAHUJA, José Antonio. (2009), “Del 'Regionalismo Abierto' al 'Regionalismo Post-Liberal'. Crisis y Cambio en la Integración Regional en América Latina”. Anuario de la Integración Regional de América Latina y el Gran Caribe 2008-2009. Coordinadora Regional de Investigaciones Económicas y Sociales (CRIES), pp. 11-54.

SECRETARÍA DE ECONOMÍA. (2013), "Tratados y Acuerdos Firmados por México" (capturado el 15 de agosto de 2013 en http://www.economia.gob.mx/comunidad-negocios/comercio-exterior/tlc-acuerdos).

SELA. (2010), “Evolución Reciente de las Relaciones Económicas entre la República Popular China y América Latina y el Caribe. Mecanismos Institucionales y de Cooperación para su Fortalecimiento". SELA, Caracas, Venezuela, octubre, pp. 1-39.

SEVERINO, Rodolfo. (2007), “Integración Económica Regional en Asia-Pacífico: La Experiencia de ASEAN". Anuario de Asia Pacífico 2007, 22 de febrero, pp. 301-310.

XINHUA.NET (2007), "ASEAN y EEUU Firmarán TAC esta Semana”, 20 de julio (capturado el 1 de julio de 2012 en http://www.spanish.xinhuanet.com/spanish/ 2009-07/20/content_908766.htm). 


\title{
RESUMO
}

Novas Configurações Econômicas no Eixo Ásia-Pacífico e suas Consequências para a América Latina: Da APEC à Aliança do Pacífico

Este artigo analisa as novas tendências na configuração dos espaços de cooperação econômica na região Ásia-Pacífico que se estabelecem em 2004 e se expressam no Acordo Estratégico Trans-Pacífico de Associação Econômica, o Acordo de Associação Trans-Pacífico, a Área de Livre Comércio da Associação de Nações do Sudeste Asiático com a China, o Japão e a Coreia do Sul, e a Aliança do Pacífico. Argumenta-se que nesta nova configuração de sub-regiões estratégicas produz-se uma dupla dinâmica: por um lado, uma maior cooperação econômica é impulsionada para conformar uma área de livre-comércio regional na Bacia do Pacífico e, por outro, ativam-se dinâmicas de rivalidade em função dos interesses de potências como os Estados Unidos e a China nestas sub-regiões. Finalmente, examina-se a participação de alguns países latinoamericanos, como Peru, Chile, México e Colômbia, nestes acordos.

Palavras-chave: Ásia-Pacífico; cooperação econômica; América Latina; Aliança do Pacífico

\begin{abstract}
New Economic Configurations in Asia-Pacific and its Consequences for Latin America: From APEC to the Pacific Alliance

This article analyzes the emergent trends that have been shaping economic cooperation in the Asia-Pacific region since 2004 resulting in the Trans-Pacific Strategic Economic Partnership, the Trans-Pacific Partnership Agreement, the Free Trade Area Association of Southeast Asian Nations (China, Japan and South Korea), and the Pacific Alliance. This new configuration of strategic sub-regions has a double-sided dynamic: on one hand, a push for greater economic cooperation fostering regional free trade area in the Pacific Rim, and, second, the promotion of rivalries in these sub-regions according to the interests of the major powers in the region such as the United States and China. It also examines the participation of Latin American countries such as Peru, Chile, Mexico and Colombia in these agreements.
\end{abstract}

Keywords: Asia-Pacific; economic cooperation; Latin America; Pacific Alliance 


\section{RÉSUMÉ}

Nouvelles Configurations Économiques dans l'Axe Asie-Pacifique et leurs Conséquences pour l'Amérique Latine: De l' APEC à L'Alliance du Pacifique

Dans cet article, on examine les nouvelles tendances dans la configuration des espaces de coopération économique dans la région Asie-Pacifique qui se dessinent en 2004 et s'expriment dans l'Accord Estratégique Trans-Pacifique d'Association Economique, l'Accord d'Association Trans-Pacifique, la zone de libre commerce de l'Association de Nations du Sud-Est Asiatique avec la Chine, le Japon et la Corée du Sud, et l'Alliance du Pacifique. On soutient que, dans cette nouvelle configuration de sous-régions stratégiques, il se produit une double dynamique: d'un côté, une plus grande coopération économique est stimulée pour former une zone de libre commerce régional dans le Bassin du Pacifique et, d'un autre, des dynamiques de rivalité se mettent en œuvre en fonction des intérêts de puissances telles que les États-Unis et la Chine dans ces sous-régions. On conclut par l'examen de la participation de certains pays latino-américains, tels que le Pérou, le Chili, le Mexique et la Colombie, dans ces accords.

Mots-clés: Asie-Pacifique; coopération économique; Amérique Latine; Alliance du Pacifique

\section{RESUMEN}

Nuevas Configuraciones Económicas en el Asia-Pacífico y sus Consecuencias para América Latina: Desde el APEC a la Alianza del Pacífico

Este artículo analiza las nuevas tendencias en la configuración de los espacios de cooperación económica en la región del Asia-Pacífico que se establecen desde 2004 y se expresan en el Acuerdo Transpacífico de Asociación Económica Estratégica, el Acuerdo de Asociación Transpacífico, el Área de Libre Comercio de la Asociación de Naciones del Sudeste Asiático con China, Japón y Corea del Sur, y la Alianza del Pacífico. Se argumenta que, en esta nueva configuración de subregiones estratégicas, se produce una doble dinámica: por un lado, se impulsa una mayor cooperación económica que permita conformar un área de libre comercio regional en la Cuenca del Pacífico y, por otro, se activan dinámicas de rivalidad en función de los intereses de las principales potencias en la región como son Estados Unidos y China en relación con estas subregiones. También se examina la participación de países latinoamericanos como Perú, Chile, México y Colombia en estos acuerdos.

Palabras claves: Asia-Pacífico; cooperación económica; América Latina; Alianza del Pacífico 